\title{
Medición de nivel de cultura ergonómica en supervisores y alta dirección en una empresa manufacturera.
}

\section{Ergonomic culture level measurement in supervisors and senior management in a manufacturing company}

\author{
Allán Chacara Montes ${ }^{1}$, Mauricio López Acosta ${ }^{2}$, José Manuel Velarde Cantú $^{3}$
}

${ }^{1}$ Departamento de Ingeniería Industrial y de Sistemas, Instituto Tecnológico de Sonora, ITSON, Navojoa, Son. México. E-mail: allan.chacara@itson.edu.mx

${ }^{2}$ Departamento de Ingeniería Industrial y de Sistemas, Instituto Tecnológico de Sonora, ITSON, Navojoa, Son. México. E-mail: mauricio.lopez@itson.edu.mx

${ }^{3}$ Departamento de Ingeniería Industrial y de Sistemas, Instituto Tecnológico de Sonora, ITSON, Navojoa, Son. México. E-mail: jose.velarde@itson.edu.mx

DOI: $\underline{\text { https://doi.org/10.46589/rdiasf.vi36.406 }}$

Recibido 4 de junio 2021.

Aceptado 30 de septiembre 2021

Publicado 1 de noviembre 2021

\section{Resumen.}

El siguiente proyecto tiene como propósito medir el nivel de cultura ergonómica de una empresa manufacturera que se dedica a la elaboración de cables, fibra óptica, sistemas de cableados y servicio relacionados para el sector automotriz y sistemas de cable; para realizar dicho estudio se utilizó como herramienta el cuestionario de cultura ergonómica en centros de trabajo (CCE-T) y fue aplicado a una muestra de 26 personas entre supervisores y gerentes. Este cuestionario permitió medir el nivel de desarrollo de la cultura ergonómica en centros de trabajo, desde la óptica de los que supervisores y la alta dirección (gerentes) lo cual permitió obtener una percepción que tienen sobre el desarrollo y aplicación de la ergonomía en su empresa y poder tener una visión más amplia y poder obtener una retroalimentación para que la empresa pueda obtener un alto grado de cultura ergonómica. Por consiguiente, este instrumento se considera un elemento valioso como mecanismo de realimentación de procesos en salud colectiva y como herramienta diagnóstica.

Palabras clave: Cultura Ergonómica, Dirección, Evaluación. 


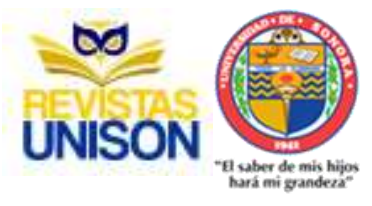

\begin{abstract}
The purpose of the following project is to measure the level of ergonomic culture of a manufacturing company that is dedicated to the development of cables, fiber optics, cabling systems and related services for the automotive sector and cable systems; To carry out this study, the ergonomic culture questionnaire in work centers (CCE-T) was used as a tool and it was applied to a sample of 26 people between supervisors and managers. This questionnaire made it possible to measure the level of development of ergonomic culture in work centers, from the perspective of supervisors and senior management (managers), which allowed them to obtain a perception they have about the development and application of ergonomics in their company and to be able to have a broader vision and be able to obtain feedback so that the company can obtain a high degree of ergonomic culture. Consequently, this instrument is considered a valuable element as a feedback mechanism for collective health processes and as a diagnostic tool.
\end{abstract}

Keywords: Ergonomic Culture, Direction, Evaluation.

\title{
Introducción
}

La industria automotriz es de alto volumen de producción, eso significa que se ensamblan componentes a una velocidad de 50 segundos por estación de trabajo y aun cuando esta industria es de base tecnológica, persisten partes del proceso donde los ensambles son manuales o de manera casi artesanal. Haciendo los cálculos con base a esos datos, y asumiendo que cada cinco segundos se ensambla o se utiliza un componente, en total: cada segundo se utilizaría 18 millones de componentes, solo para la industria en México, esto se puede interpretar como que cada segundo se mueve 18 millones de veces las extremidades superiores en los trabajadores; dedos, manos, codos, hombros, cuello y espalda (Millán, 2016).

La Ergonomía en las empresas del sector de la automoción, goza de muchos años de implantación y desarrollo continuo a escala mundial, todo y que nuestra experiencia nos apunta que los recursos que se destinan, know-how y estándares de las empresas establecidas en Europa, aunque por delante del que vemos en empresas Latino Americanas, se basa en modelos de gestión corporativos que no siempre 
generan el impacto esperado.

Hablamos de un sector con altos niveles de exigencia productiva, que precisa de realizar cambios constantes en las líneas de producción y tecnología, y donde el alto coste unitario y precio de venta de su producto, hace viable la inversión de importantes recursos en nueva tecnología y sistemas que mejoren los riesgos ergonómicos (CENEA, 2019).

Ardila \& Rodríguez, (2016) tras una revisión de la literatura, realizaron un constructo teórico fundamentado en el modelo de Scott, Kogi y McPhee y que contempla las dimensiones macroergonomía y microergonomía y los componentes diseño organizacional, diseño de instalaciones, entrenamiento/educación, puesto de trabajo, diseño de la tarea, equipos/productos y herramientas. A partir de este, se elaboraron un cuestionario de 27 puntos con respuestas tipo Likert. Las preguntas se perfilaron de manera que pudieran ser resueltas en cualquier contexto empresarial. Se generó un primer borrador con cada uno de los ítems del cuestionario, que fue revisado por cinco expertos en ergonomía, los cuales hicieron ajustes de pertinencia y adecuación. Se asignó la denominación definitiva del instrumento como: Cuestionario de Cultura Ergonómica en Centros de Trabajo (CCE-T).

Para la asignación de valores las categorías tomadas de la escala deLikert planteadas en el cuestionario llevan al evaluador a ponderar cada ítem. Así, el total de las respuestas totalmente de acuerdo se multiplican por uno; parcialmente de acuerdo, por dos; parcialmente en desacuerdo, por tres; y totalmente en desacuerdo, por cuatro. Para llevar el total a una clasificación, a partir de los resultados se tomó el total de la sumatoria de respuestas de cada una de las cuatro categorías, y se trasladó a una tabla de ponderados que permiten, finalmente, dar el resultado del nivel en el que el trabajador clasifica a su empresa.

Al evaluar el nivel de cultura ergonómica, se espera que una empresa pueda desarrollar, los autores han definido cuatro niveles de cultura ergonómica: baja, media, alta y muy alta, producto de la sumatoria de los totales parciales ya ponderados. Esta categorización permite orientar a la empresa sobre lo que consideran sus trabajadores en cuanto a actuaciones en ergonomía y comparar los resultados con lo existente (Jaimes, 2018).

En resumen, el fin que la ergonomía persigue es que los empleados se encuentren cómodos en sus lugares de trabajo. Este objetivo no supone solo una premisa ética, sino que también va a tener que ver con la optimización de los procesos habituales de una empresa. Si en una firma se dan las condiciones precisas para que sus trabajadores se encuentren cómodos en sus puestos, un incremento de su 
rendimiento llevará al aumento de la productividad. La firma será, en consecuencia, la principal beneficiada de la implementación de las medidas ergonómicas. Implicarán una primera inversión que será rentabilizada con el paso del tiempo (ARSAM, 2020).

Algunas revisiones han aportado datos sobre los beneficios que conllevan las intervenciones en ergonomía a dichos factores, subrayando la importancia de incluir en estas estrategias de intervención, no solo a los trabajadores, sino a los supervisores y a la alta dirección. Dicho esto, se espera entonces que una empresa desarrolle un alto grado de cultura ergonómica, como piedra angular de lo que se considera la intervención primaria en ergonomía (Guzmán, 2010).

El término cultura ergonómica se entiende como "el conjunto de formas y expresiones aplicadas para trasformar el trabajo, que caracterizan a una organización" por tanto, para que exista una verdadera cultura ergonómica se requiere, entre otros, de un "ambiente favorable, un pensar colectivo y de la reafirmación social de las necesidades en los lugares de trabajo" (Gómez, 2002).

El objetivo del presente estudio es determinar el nivel de cultura que se tiene en supervisores y gerentes en una empresa manufacturera y para ello se ha llevado a cabo la aplicación de un cuestionario para medir la cultura ergonómica en los supervisores y gerentes en una empresa manufacturera.

\section{MATERIALES Y MÉTODOS}

\section{A. Sujeto de Estudio}

Los sujetos de estudio fueron los supervisores y gerentes de línea de una empresa manufacturera. Para la aplicación de cuestionario se tomó en cuenta que la empresa cuenta con 21 supervisores y 7 gerentes en todas sus áreas desde prototipo, preparación, comax, producción y moldeo y para realizar estudio se hizo un cálculo del tamaño de muestra mediante una herramienta estadística, para conocer el número de cuestionarios a aplicar con el objetivo de medir el nivel de cultura ergonómica en la empresa.

Para el tamaño de muestra se tomó en cuenta que es una muestra finita, con una probabilidad de éxito de $95 \%$, un nivel de confianza de 5\%, el cálculo se realizó en software (Excel) y por lo tanto el tamaño de muestra fue de 26 supervisores y gerentes a los cuales se les aplicará el cuestionario con el objetivo de conocer su percepción de sobre el desarrollo y aplicación de la ergonomía. 
B. Procedimiento

A continuación, se describirá los pasos que se llevaron a cabo para la aplicación del cuestionario de cultura ergonómica en centros de trabajos CCE-T.

- Confiabilidad del instrumento

En esta actividad se determinó la confiabilidad del instrumento determinando el Alfa de Cronbach.

- Aplicación de cuestionario.

En esta actividad se aplicó un cuestionario a los supervisores y gerentes de la empresa para conocer su percepción sobre el desarrollo y aplicación de la ergonomía.

- Análisis de resultados.

De acuerdo a la recolección de datos, se analizarán las respuestas para conocer el nivel de cultura ergonómica con la que cuenta la empresa.

C. Materiales

Los materiales utilizados para llevar a cabo el proyecto fueron los siguientes:

- Software SPSS.

- Cuestionario de cultura ergonómica en centros de trabajos CCE-T de (Jaimes \& Rodríguez, (2016).

\section{Resultados y su discusión}

A continuación, se presentarán los resultados más relevantes que arrojo el proyecto después de las aplicaciones del instrumento para media la cultura ergonómica en los centros de trabajo.

\section{Participantes.}

El instrumento aplicado cuenta con 26 elementos y nos arroja una confiabilidad alta con un coeficiente Alfa Cronbach de 0.897, la muestra $(n=26)$ se conformó principalmente por hombres 16 
Hombres $(61,5 \%)$ y 10 mujeres $(38,5 \%)$. Su edad promedio fue 34,4 años y registraron niveles de escolaridad altos (Licenciatura y Maestría). Llevan en promedio 3,2 años laborando en la empresa.

El primer resultado relevante de este estudio se obtuvo mediante la sumatoria del nivel de cultura ergonómica que tiene cada que uno de los sujetos de estudio obteniendo un puntaje de 52.1 pts., según la escala utilizada de 40-52 es Alta y de 53-78 es Media, y se procedió a agruparlos según nivel obteniendo un 54\% de los participantes nivel medio y un $46 \%$ nivel alto, ver figura 1.

\section{Figura 1.}

Nivel de CCE por categoría.

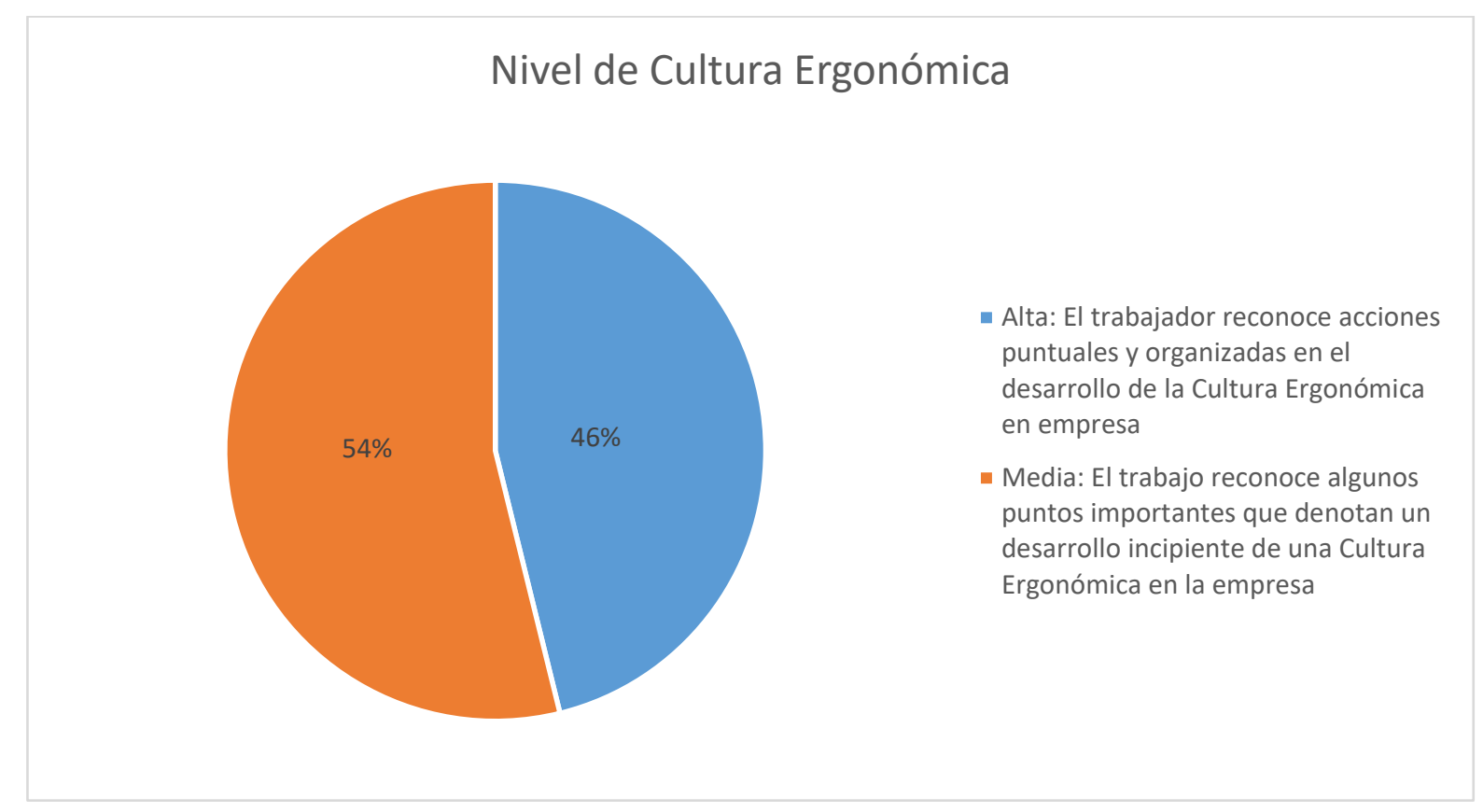

Análisis por pregunta de los resultados más relevantes del estudio:

Pregunta número cuatro del cuestionario: “¿Se han realizado mediciones de las partes de su cuerpo para hacer ajustes a su puesto de trabajo durante el tiempo que lleva laborando en esta empresa?" presenta un resultado que muestra que el 100\% está en "Totalmente en desacuerdo" como se muestra en la Figura 2, esto indica que su estación de trabajo o herramientas de trabajo no están debidamente ajustadas a sus medidas antropométricas. 


\section{Figura 2.}

Porcentaje de personas que han sido medidas para su área de trabajo

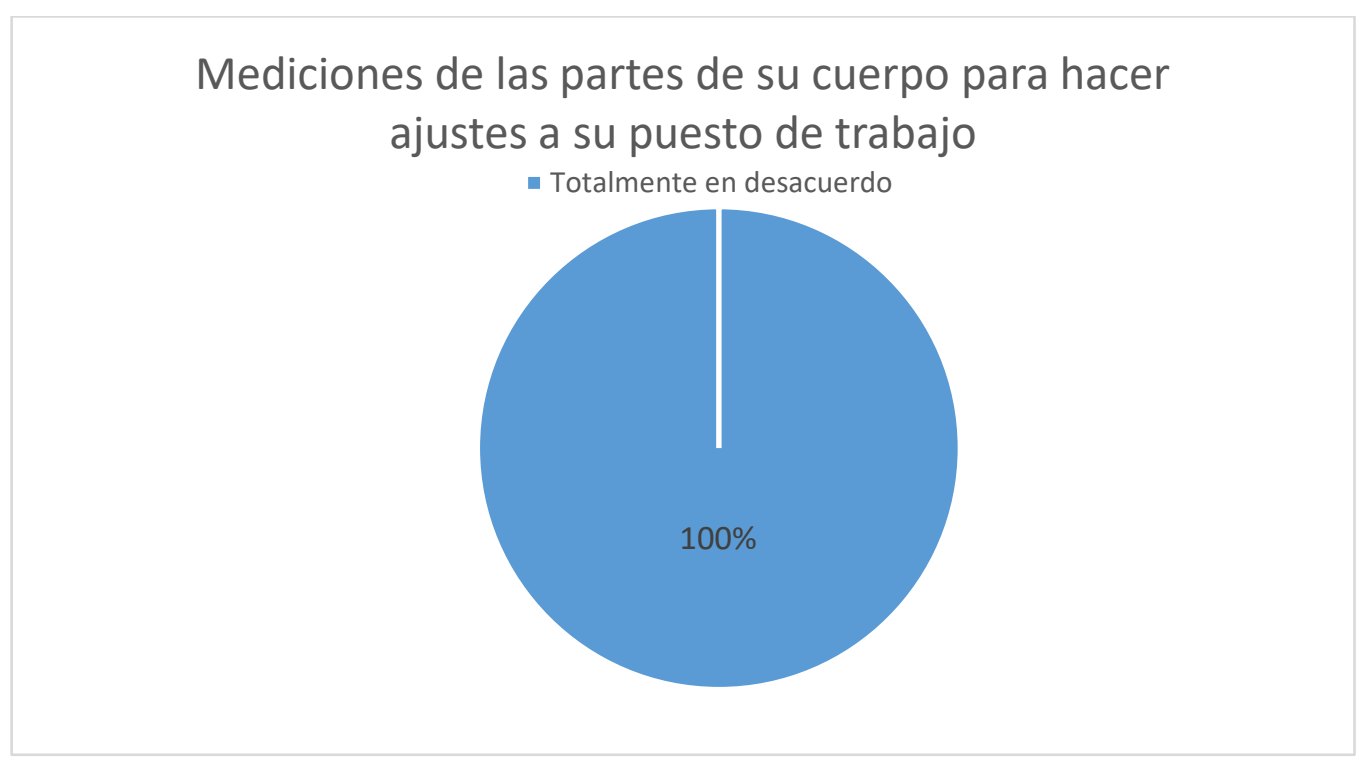

Pregunta número once del cuestionario: ¿Ha recibido reinducción de la labor que realiza?” presenta un resultado que muestra que el 50\% está en "Parcialmente en desacuerdo" y el otro 50\% esta "Parcialmente de acuerdo" como se muestra en la Figura 3, esto indica que solo la mitad de los sujetos bajo estudio han recibido alguna reinducción en la labor que realiza en la empresa.

\section{Figura 3.}

Porcentaje de reinducción de la tarea realizada

¿Ha recibido reinducción de la labor que realiza?

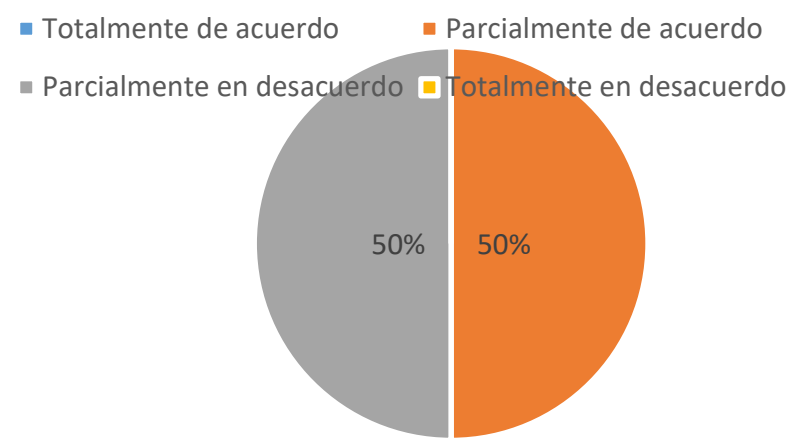


Pregunta número doce del cuestionario: “¿Recibe entrenamiento para mejorar la labor que realiza?” presenta un resultado que muestra que el 61\% está en "Parcialmente en desacuerdo", el 31\% esta " Parcialmente de acuerdo" y el 8\% está "Totalmente de acuerdo" como se muestra en la Figura 4, esto dice que su la mayoría de los sujetos bajo estudio (61\%) no recibe un entrenamiento adecuado para mejorar la labor que realiza mientas que el otro (39\%) recibe un entrenamiento adecuado o regular para mejorar la labor que realiza.

\section{Figura 4.}

Porcentaje de entrenamiento para mejorar la tarea realizada

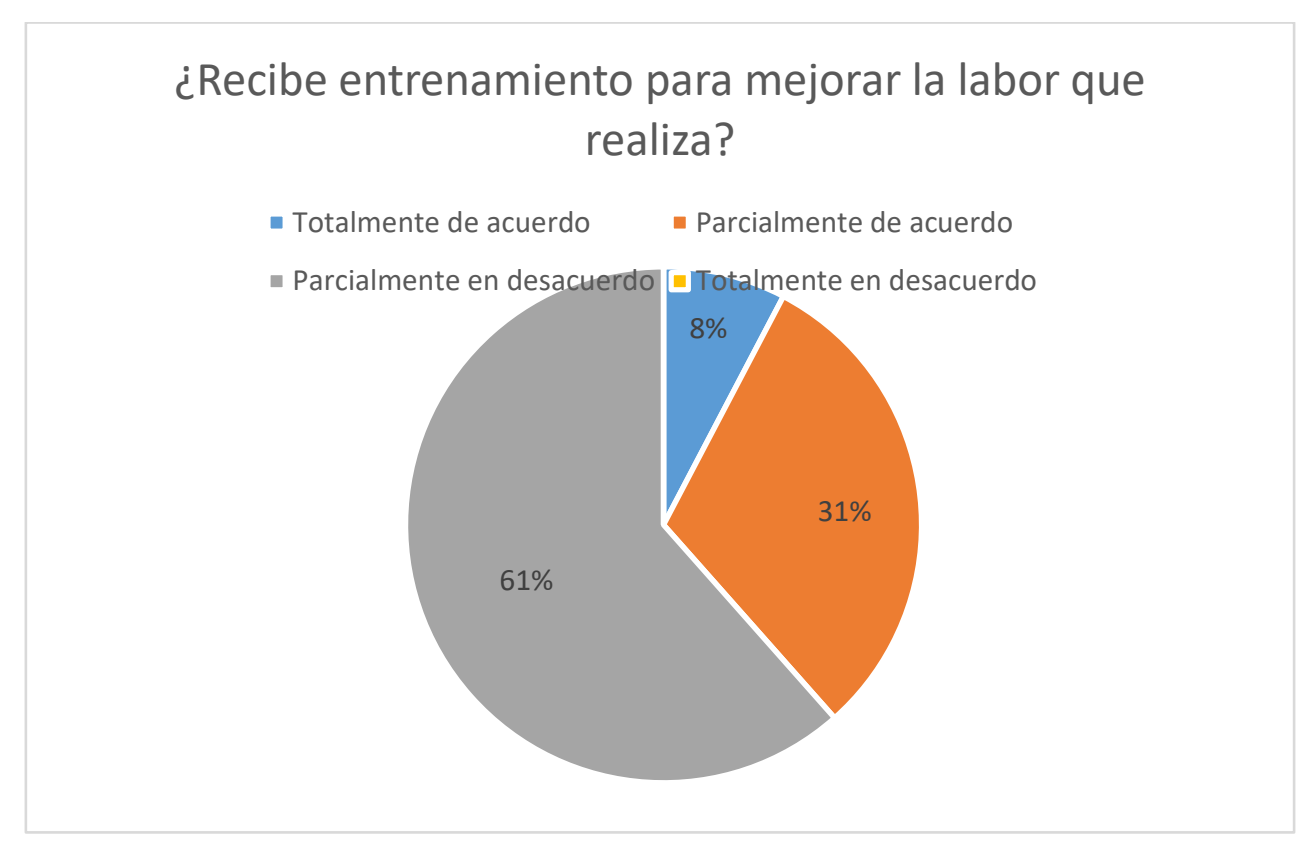

Pregunta número diecisiete del cuestionario: “¿Se realizan evaluaciones de las condiciones en las que se encuentra su espacio físico de trabajo?” presenta un resultado que muestra que el 69\% está en "Parcialmente de acuerdo" y el 31\% se muestra "Parcialmente en desacuerdo" como se muestra en la Figura 5, esto indica que no se realiza de manera frecuente o regular evaluaciones sobre las condiciones del espacio físico de trabajo de los sujetos bajo estudio. 


\section{Figura 5.}

Porcentaje de personas que han sido consideradas, para mejorar su área de trabajo

$$
\begin{gathered}
\text { ¿Se realizan evaluaciones de las condiciones en las que se } \\
\text { encuentra su espacio físico de trabajo? } \\
\text { - Totalmente de acuerdo } \quad \text { - Parcialmente de acuerdo } \\
\text { - Parcialmente en desacuerdo } \quad \text { - Totalmente en desacuerdo }
\end{gathered}
$$

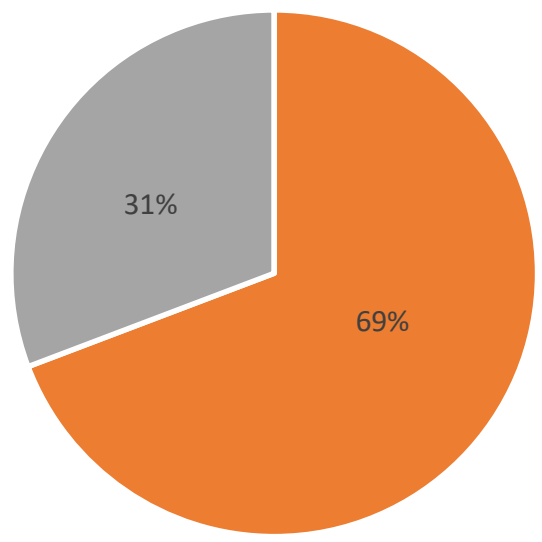

Pregunta número dieciocho del cuestionario: ¿Se han hecho cambios en las tareas que usted realiza de manera que le generen menos riesgo de enfermedad o lesión?” presenta un resultado que muestra que el 69\% está en "Parcialmente de acuerdo", el 23\% esta "Parcialmente en desacuerdo" y el $8 \%$ está "Totalmente de acuerdo" como se muestra en la Figura 6, esto nos indica que en la mayoría de los sujetos bajo estudio se han realizado cambios en la tareas que realizan de manera regular cambios en las tareas que realizan de manera que generan menos riesgo de enfermedad o lesión mientras que tan solo el $23 \%$ arroja que no se realizan cambios en la tareas de manera regular. 


\section{Figura 6.}

Porcentaje de trabajadores que encuentran menor riesgo en la tarea realizada

\section{¿Se han hecho cambios en las tareas que usted realiza de} manera que le generen menos riesgo de enfermedad o lesión?

\footnotetext{
- Totalmente de acuerdo $\quad$ - Parcialmente de acuerdo

- Parcialmente en desacuerdo - Totalmente en desacuerdo
}

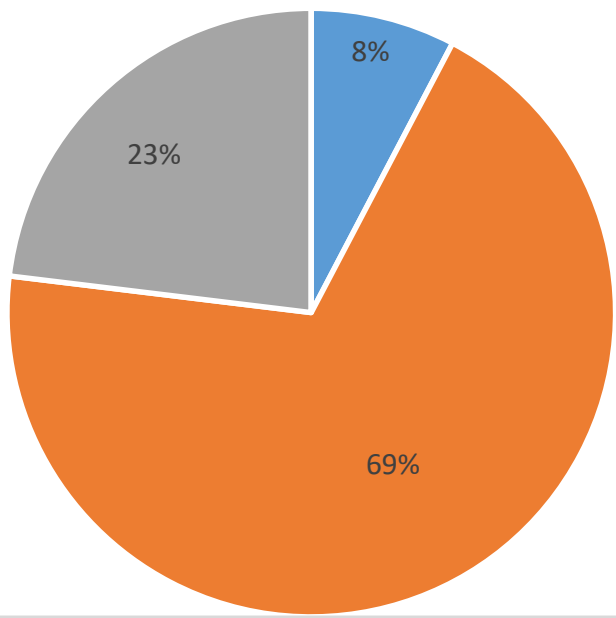

Pregunta número veinte del cuestionario: “¿Se han hecho ajustes a las funciones y tareas en su puesto en pro de disminuir el riesgo de enfermedad o accidente?" presenta un resultado que muestra que el 65\% está "Parcialmente de acuerdo", El 27\% esta "Parcialmente en desacuerdo" y el 8\% está "Totalmente de acuerdo" como se muestra en la Figura 7, esto nos dice que su la mayoría de los sujetos bajo estudio (73\%) se han hecho ajustes a las funciones y tareas en su puesto en pro de disminuir el riesgo de enfermedad o accidente mientras que el $27 \%$ no se han hecho ajustes a sus funciones o tareas de manera regular en su puesto en pro de disminuir el riesgo de enfermedad o accidente. 


\section{Figura 7.}

Porcentajes de percepción de riesgo o enfermedad del trabajador en el área de trabajo

¿Se han hecho ajustes a las funciones y tareas en su puesto en pro de disminuir el riesgo de enfermedad o accidente?

- Totalmente de acuerdo

- Parcialmente de acuerdo

- Parcialmente en desacuerdo

- Totalmente en desacuerdo

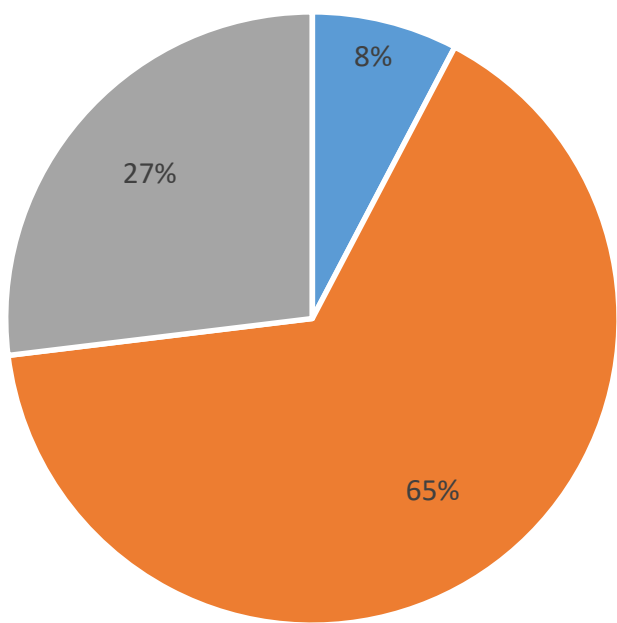

Pregunta numero veintidós del cuestionario: “¿Se han hecho evaluaciones para determinar la necesidad de utilizar elementos que faciliten el trabajo que usted realiza?" presenta un resultado que muestra que el 54\% está en "Parcialmente en desacuerdo" y el otro 46\% está "Totalmente en desacuerdo" como se muestra en la Figura 8, esto nos indica que más de la mitad de los sujetos bajo estudio no se le ha hecho alguna evaluación para determinar si tienen la necesidad de utilizar elementos que faciliten el trabajo que realizan mientras que al restante $(46 \%)$ de manera nula se le ha hecho alguna evaluación para determinar la necesidad de utilizar elementos que faciliten el trabajo que realizan. 
Figura 8.

Porcentaje de trabajadores que no han sido evaluados para facilitar o reducir el riesgo

En tareas realizadas.

¿Se han hecho evaluaciones para determinar la necesidad de utilizar elementos que faciliten el trabajo que usted realiza?

- Totalmente de acuerdo

- Parcialmente de acuerdo

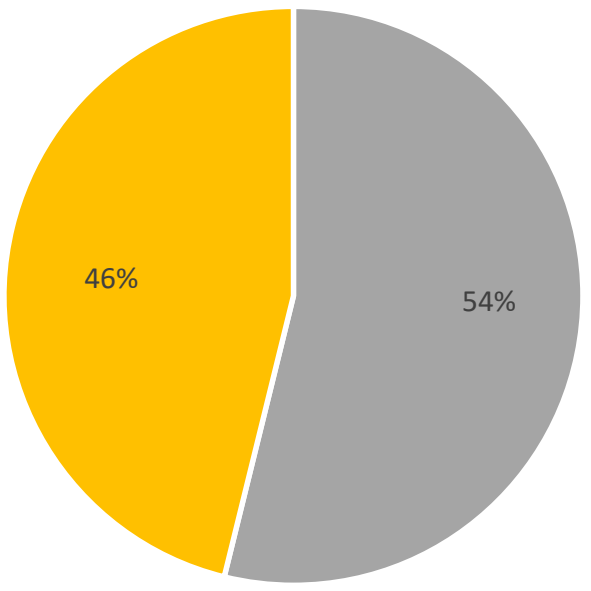

- Parcialmente en desacuerdo

- Totalmente en desacuerdo

Pregunta número veintitrés del cuestionario: ¿Utilizan elementos que faciliten el trabajo que usted realiza?" presenta un resultado que muestra que el 96\% está en "Totalmente desacuerdo" mientras que el 4\% esta "Parcialmente en desacuerdo" como se muestra en la Figura 9, esto nos indica que gran parte casi en su totalidad (96\%) de los sujetos bajo estudio no se utilizan elementos que faciliten el trabajo que realizan mientras que el restante $(4 \%)$ se han utilizado de manera muy escaza elementos que les ayuden a facilitar el trabajo que realizan. 


\section{Figura 9.}

Porcentaje de trabajadores que utilizan una herramienta de apoyo en sus labores

$$
\text { ¿Utilizan elementos que faciliten el trabajo que usted realiza? }
$$

- Totalmente de acuerdo - Parcialmente de acuerdo $\quad$ Parcialmente en desacuerdo $\square$ Totalmente en desacuerdo

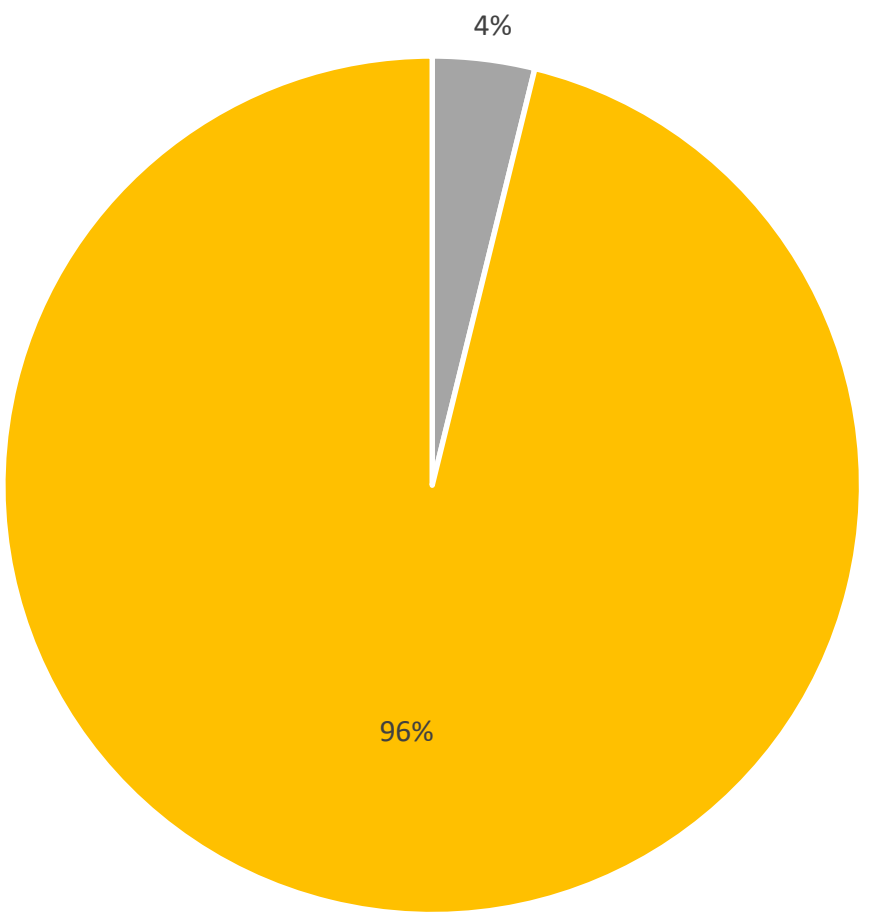

\section{Conclusiones y recomendaciones}

Como conclusión sobre este proyecto es que los resultados en general sobre la cultura ergonómica dieron un promedio de una calificación "Alta” en cultura ergonómica en supervisores y gerentes en la empresa, pero no por ello la empresa no debe realizar diversos ajustes al momento de realizar un diagnóstico o 
evaluación ergonómica si las estaciones o las diversas herramientas que utiliza el personal son las aptas para desarrollar sus actividades de manera que sea mínimo el riesgo de que el empleado sufra alguna enfermedad de trastorno musculo-esquelético (TME) o algún accidente de trabajo.

Como recomendación hacia la empresa tomando en cuenta estos resultados el primero sería como el más crítico el realizar un estudio antropométrico en la empresa ya que el resultado dado en la parte de si se han tomado en cuenta las dimensiones de su cuerpo al momento de hacer ajustes a su puesto de trabajo el resultado fue $100 \%$ que no se les ha realizado algún estudio antropométrico y ese es un aspecto que hay que acatar ya que de ahí puede ser el origen de muchos problemas que podrían estar sufriendo actualmente algunos trabajadores o en un futuro a corto plazo podrían empezar a tener problemas físicos debido a que su puesto de trabajo o herramientas no están debidamente adaptados a sus medidas antropométricas.

Realizar con mayor frecuencia o de manera más regular las reinducciones sobre la labor que realiza el personal ya que quedó demostrado que el $50 \%$ no recibe reinducciones en sus labores de manera regular o de una manera no del todo satisfactoria.

Llevar a cabo mayor capacitaciones o entrenamientos ya que más de la mitad de los sujetos bajo estudio mostraron un descontento en cuanto a los entrenamientos o capacitaciones que se les brinda para mejorar su rendimiento al momento de realizar sus labores.

Tratar de incrementar el número de evaluaciones que se le realiza al espacio de trabajo donde realiza sus labores ya que, aunque el resultado arrojo que tiene un grado aceptable este puede ser aún mayor y que este incremento genere como consecuencia un mayor rendimiento del personal al momento de desarrollar sus labores.

De igual manera que en la parte de evaluaciones, el tratar de realizar un mayor número de cambios en las tareas que el personal realiza de tal manera que le generen menos riesgo de enfermedad o lesión, aunque la aprobación de estos fue aceptable esta puede ser aún mayor que la que se obtuvo. Otra de los resultados que van de la mano con el anterior es sobre si han hecho ajustes a las funciones y tareas en su puesto en pro de disminuir el riesgo de enfermedad o accidente, en resultado fue aceptable, aunque igual que el anterior este puede ser a un más favorable mediante evaluaciones y ajustes en las funciones del personal para disminuir de manera constante el riesgo de una enfermedad de trabajo algún accidente.

Un resultado muy importante a resaltar es la evaluación y utilización de elementos que faciliten el trabajo que el personal realiza ya que la gran parte de los sujetos bajo estudio arrojo un muy bajo índice 
en cuanto a la realización de evaluaciones sobre la necesidad de utilizar elementos que faciliten el trabajo del personal, como elementos que se podría utilizar serian por ejemplo: apoya pies, apoya muñecas, soportes para columna vertebral, descansa pies, soporte para portátiles, entre otros. También se recomienda estar realizando constantemente las inspecciones a las áreas, equipos e instalaciones buscan identificar el mayor número de condiciones que representen mayor riesgo para su seguridad y salud en el lugar de trabajo.

\section{REFERENCIAS}

ARSAM. (2020, 2 enero). "5 buenos ejemplos de la ergonomía en la industria automotriz | ARSAM". ARSAM | Herramientas industriales. https://www.arsam.es/5-buenos-ejemplos-de-laergonomia-en-la-industria-automotriz/

CENEA. (s. f.). Riesgos Ergonomicos Sector Automotriz | Cenea. CENEA La Ergonomía Laboral del S.XXI. Recuperado 27 de octubre de 2020, de https://www.cenea.eu/consultoria-ergonomiaempresas/sectores/ergonomia-industrial-automocion-automotriz/

Guzman E., Saenz Zapata LM. (2010,10 noviembre). La ergonomía en el contexto de la prevención. Realidad y propuestas educativas. Memorias: 16a Semana de la Salud Ocupacional y 9o Congreso Colombiano de Ergonomía. Medellín (Colombia).

Gómez-Conesa A., Martínez- González M. Ergonomía. Historia y ámbitos de aplicación, Fisioterapia. 2002;24(1):3-10.

Jaimes, C. P. A., \& Rodríguez, R. M. R. A. (2018). Validación de un cuestionario de cultura ergonómica en centros de trabajo CCE-T | Revista Investigaciones Andina. Investigaciones Andina. https://revia.areandina.edu.co/index.php/IA/article/view/98 
Millán, D. F. (2016,14 Noviembre). Proyecto Puente. "La importancia de la ergonomía en la salud ocupacional": https://proyectopuente.com.mx/2016/11/14/la-importancia-la-ergonomia-en-lasalud-ocupacional/

Cómo citar:

Chacara Montes, A. ., López Acosta, M. ., \& Velarde Cantu, J. M. (2021). Medición de nivel de cultura ergonómica en supervisores y alta dirección en una empresa manufacturera. Revista De Investigación Académica Sin Frontera: División De Ciencias Económicas Y Sociales, (36). https://doi.org/10.46589/rdiasf.vi36.406
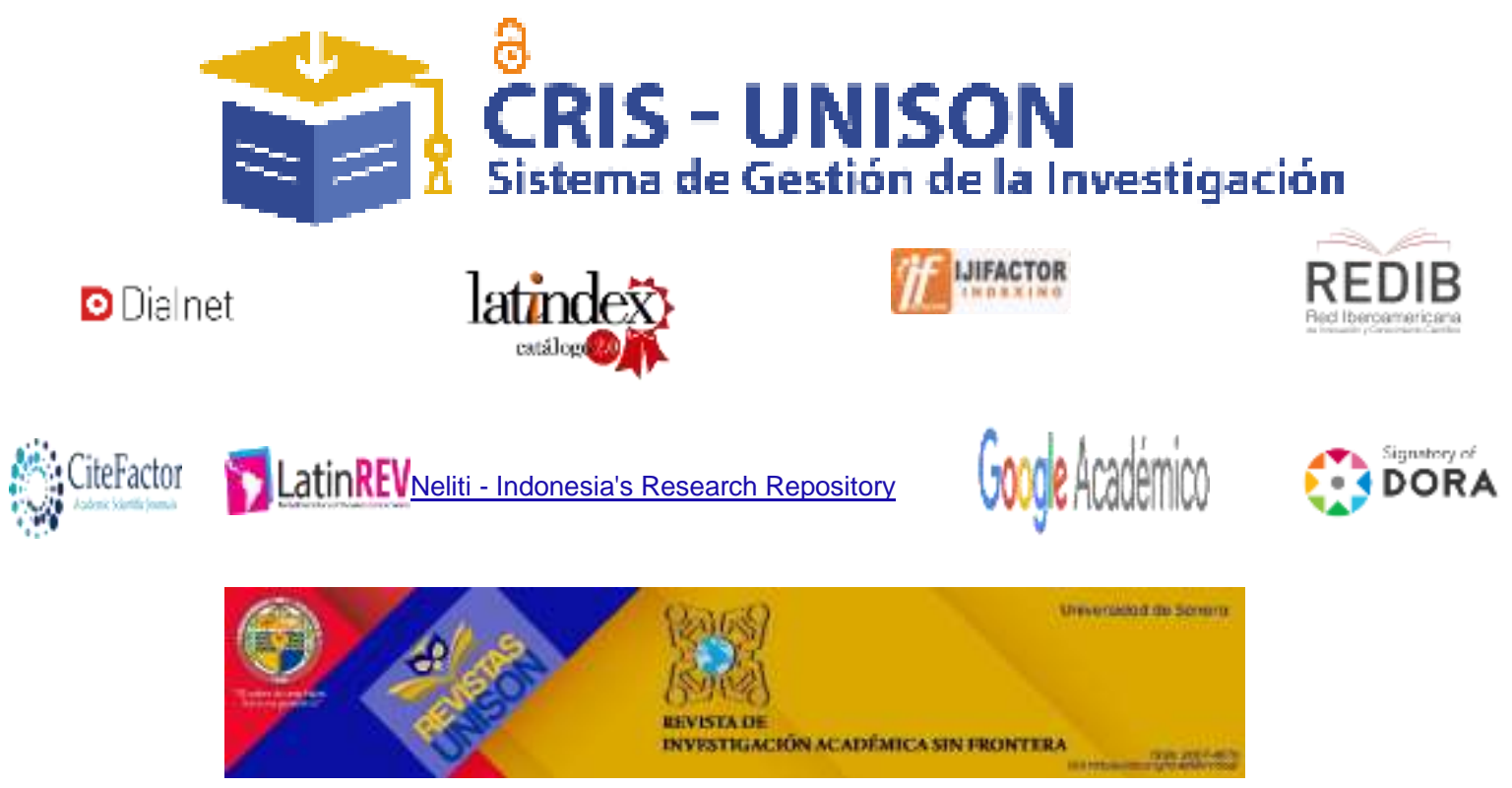